G. McHale and M.I. Newton, Global geometry and the equilibrium shapes of liquid drops on fibers, Colloids and Surfaces, A206 (103) (SI) (2002) 79-86.

\title{
Global Geometry and the Equilibrium Shapes of Liquid Drops on Fibers
}

\author{
G. $\mathrm{M}^{\mathrm{c}} \mathrm{Hale}{ }^{\star}$ and M.I. Newton \\ Department of Chemistry and Physics \\ The Nottingham Trent University \\ Clifton Lane \\ Nottingham NG11 8NS, UK.
}

\begin{abstract}
The wetting of a planar surface depends upon both the chemical nature of the surface and the local geometry. On a chemically identical solid surface in the form of a fiber the wetting is significantly different due to the global geometry of the cylindrical shape. A fluid that fully wets a material in the form of a smooth planar surface may not wet the same material when presented as a smooth fiber surface. On a fiber, a vanishing contact angle is not a sufficient condition for the formation of a wetting film; a macroscopic barrel shaped droplet with a vanishing equilibrium contact angle can exist. Moreover, two distinctly different geometric shapes of droplet are possible: a barrel and a clam-shell. In this work, these two shapes are considered using an analytical result for the barrel shape and a finite element calculation for the clam-shell shape. The surface free energies for these two conformations are evaluated for contact angles between $14^{\circ}$ and $70^{\circ}$ and for a wide range of droplet volumes. The results show that when the droplet volume is large or the contact angle is small, an axisymmetric barrel shape is the energetically preferred conformation, but that as the volume reduces or the contact angle increases the clam-shell shape becomes lower in energy. These results are compared to literature data for the roll-up (barrel-toclam-shell) transition and to a previously published criterion for metastability of the barrel shaped droplet. A conjecture on the role of the inflection angle in the barrel-shape droplet profile is also considered. For the contact angle range considered, the finite element results show that all barrelshaped droplets that are lower in energy than clam-shell droplets, are stable according to the metastability condition and also possess an inflection angle.
\end{abstract}

Keywords: Fiber, contact angle, wetting, stability, barrel, clam-shell, geometry, curvature.

\footnotetext{
- Corresponding author: Telephone: +44 (0)115 8483383; email: glen.mchale@ntu.ac.uk
} 


\section{Introduction}

The wetting of surfaces involves both surface chemistry and geometry. Geometry can be either local, in the form of rough [1,2] or patterned [3] surfaces, or it can be global, in the form of spheres [4], cylinders/fibers [4-9], etc. Of these global geometries, cylinders are of particular importance because fibers are an extremely common material in practical applications. The study of the wetting of fibers is motivated by a range of reasons, such as fluid coating requirements [10], detergency [11], adhesion [12] and the potential importance of carbon nano-tubes [13]. It is therefore of no surprise that the measurement of contact angles on fibers and of the formation and stability of films on fibers is commonly reported. Theoretical studies of the wetting of fibers has shown that physical intuition developed from studying the wetting of flat surfaces can be inaccurate. In the case of flat surfaces, the sign of the spreading power, $S=\gamma_{\mathrm{SV}}-\left(\gamma_{\mathrm{SL}}+\gamma_{\mathrm{LV}}\right)$ where the $\gamma_{i j}$ 's are the interfacial tensions, determines whether a film forms or whether a macroscopic droplet with a non-zero contact angle exists [6]. In the fiber case, a sheathing film only forms when $S$ exceeds a finite positive value dependent on the fiber radius and a vanishing contact angle can be consistent with a macroscopic droplet $[6,7,14,15]$. In the fiber case, a zero contact angle does not imply a vanishing Laplace excess pressure as is the case for a flat surface (assuming classical capillarity) $[16,8]$. Despite our understanding of these differences between a flat surface and a fiber surface, the fact that the global geometry of a fiber leads to two fundamentally different conformations of macroscopic droplet, a barrel and a clam-shell (Figure 1), has only occasionally been studied. This appears surprising since the roll-up transition, first studied by Adam [17], is of significance in detergency [11,18] and, as we argue in this report, is of fundamental importance in understanding how a droplet-on-fiber system becomes a droplet-on-aplane surface in the limiting case of low fiber curvature. One reason for the relative neglect of the clam-shell conformation is probably the difficulty in dealing with its asymmetric shape compared to the axially symmetric barrel shaped conformation.

In this work, we consider the clam-shell droplet-on-fiber system and use finite element calculations to compute the shape of the droplets for contact angles in the range $14^{\circ}$ to $70^{\circ}$; we assume that classical capillarity holds and that gravity can be neglected. For each angle a full range of reduced droplet volume, $V_{\mathrm{r}}$, defined as the droplet volume, $V$, divided by the cube of the fiber radius, $x_{\mathrm{f}}$, is considered. The finite element results are used to estimate the surface free energy of the clam-shell shaped droplets and these energies are then compared to those of barrel shaped droplets of the same volume and with the same contact angle. The energies for the barrel shaped droplets are calculated from the known solution using elliptic integrals $[8,16]$. We are 
therefore able to present, for the first time, a "phase" diagram describing the conformation of minimum surface free energy, which we shall refer to as absolute stability, as a function of contact angle and volume. These results are compared to literature date on the roll-up transition and to theoretical predictions on the metastability of the axially symmetric barrel shape conformation [18]. A conjecture on the role of the inflection angle in the profile of the barrel drops is also considered [19]. The comparison shows that the absolute stability curve best corresponds to the experimental data. The results also show that barrel shaped droplets with surface free energy less than that of clam-shell shaped droplets (i.e. possessing absolute stability) are also stable, according to Carroll's metastability criterion [18], and, furthermore, possess inflections in their profiles.

\section{Barrel droplet-on-fiber}

In the absence of gravity the equilibrium shape of a drop surface is that the Laplace excess pressure, $\Delta P$, across the drop surface is everywhere constant,

$$
\Delta P=\gamma_{L V}\left(\frac{1}{R_{1}}+\frac{1}{R_{2}}\right)=\text { constant }
$$

where $R_{1}$ and $R_{2}$ are the principal radii of curvature at a point in the surface. Carroll [18] solved this equation for the axially symmetric barrel-shape subject to the boundary condition that the profile of the fluid surface meets the solid at an angle given by the equilibrium contact angle. The contact angle on the fiber is the same as on a flat surface and summarises the chemical nature of the surface. The solution involves elliptic integrals of the first and second kind and can be used to evaluate physical quantities, such as drop length, radius, volume, interfacial areas and Laplace excess pressure inside the drop. The solution of equation (1) enabled Carroll to develop a new drop-length - drop radius method of estimating contact angles from experimental observations of drops on fibers. This method was used in the studies of the solubilization of drops of oils on fibers that form the basis of the published data on the roll-up transition $[18,20]$.

Carroll's solution for the barrel shape droplet was subsequently used by McHale et al [8] to numerically compute the surface free energy, $F$, defined as,

$$
F=\gamma_{L V} A_{L V}+\left(\gamma_{S L}-\gamma_{S V}\right) A_{S L}
$$


where $A_{L V}$ and $A_{S L}$ are the liquid-vapor and solid-liquid interfacial areas. In the same work, the theoretical and practical importance of the inflection angle, $\theta_{1}$, was emphasised. On a flat surface, the Laplace excess pressure in a droplet can be reduced consistent with conservation of drop volume by spreading the drop into a film. At the apex of the drop the two radii of curvature in equation (1) both increase. In contrast, on a fiber, any attempt to spread the drop into a thin sheathing film necessarily requires one of these two radii of curvature to reduce in order to conserve volume; at the apex of the drop profile both radii remain positive. At the contact line, the Laplace excess pressure can be reduced by inverting the sign of one radius of curvature compared to the other. This means an inflection point can exist in the profile of a barrel-shaped droplet and this inflection may occur close to the contact line depending upon the drop volume. This is also the reason why a vanishing contact angle does not preclude a macroscopic droplet. The practical consequence of the inflection is that attempts to measure the contact angle directly by a tangent-type method may result in a measurement intermediate between the contact angle and the inflection angle. The inflection angle value depends on drop volume and can be significantly different to the true contact angle. McHale et al suggested that Carroll's method of contact angle measurement could be extended so that the inflection angle and any one other drop parameter (reduced length, $L_{r}=L_{\mathrm{W}} / x_{\mathrm{f}}$, reduced radius, $n=x_{2} / x_{\mathrm{f}}$, etc) could be used to estimate the contact angle [8]. This method was applied to experimental data using numerical routines and, subsequently, analytical approximations [8,21].

\section{Clam-shell droplet-on-fiber and surface free energy}

In contrast to the axially symmetric case, no solution to equation (1) exists for the asymmetric clam-shell shape. However, a numerical approach can be adopted using the public domain finite element Surface Evolver package [22], which was developed as part of an NSF (National Science Foundation) funded geometry supercomputing project. This method models surfaces as unions of triangles with vertices that are iteratively moved from an initial trial shape until a minimum energy configuration is obtained. McHale et al [19] have recently described the application of this package to the evaluation of the clam-shell droplet-on-fiber problem. In the present work, these calculations have been extended to larger numbers of vertices and smoother surfaces. The droplets have been evolved to their equilibrium shapes for a wide range of reduced volumes and for contact angles between $14^{\circ}$ and $70^{\circ}$ and various droplet parameters evaluated. In particular, the surface free energy of the clam-shell shapes have been compared to that for barrel shaped droplets of the same volume and with the same contact angle. The barrel shape droplet calculations of energy used the analytical solution of equation (1) rather than the finite element calculation, although tests were performed to ensure no significant difference existed with the 
finite element calculation of the barrel shapes. The results for the largest reduced volumes at which the clam-shell shape possessed a lower energy are given in table 1; this will be referred to as the absolute stability condition. Table 1 also shows the barrel drop parameters of reduced length and reduced radius. In performing the finite element calculations of the clam-shell shape it appeared that increasing the number of nodes in the mesh tended to reduce systematically the energy of the shape. Based on this observation, we conclude that clam-shells with reduced volumes below that in table 1 are likely to be of lower energy than equivalent barrel shapes, but that it is difficult to be certain that clam-shells with larger reduced volumes are definitely higher in energy than equivalent barrel-shapes. The value of reduced volume for which the barrel shape appeared to be higher in energy is shown in brackets in table 1. Two illustrative examples of clam-shell shapes with contact angles of $30^{\circ}$ and $60^{\circ}$ at the reduced volumes corresponding to the absolute stability criterion are given in figure 2. It should be noted that for presentational reasons far fewer vertices are shown than were used in the evaluation of the droplet energies.

\section{Roll-up on a fiber}

The term "roll-up" was originally used by Adam to describe the movement of a liquid off a cylinder as the contact angle increased [17]. This original view of roll-up is quite different to the increase in contact angle and reduction in contact area between a liquid drop and a plane surface. In the fiber case, if the angle is near a critical value a transformation in conformations occurs which results in further change in the contact area and the profile of the drop becomes radically different [18]. The most complete experimental data on roll-up was given by Carroll [18] and are reproduced here in figure 3 and table 2. Table 2 also includes columns for reduced volume and reduced length deduced from the literature data of contact angle and reduced maximal droplet thickness using the programs developed by McHale et al [8]. The literature data were obtained from experiments on the solubilization of an oil drop attached to a fiber immersed in a surfactant solution $[18,20]$. The drops were initially in the barrel conformation and underwent transitions to the clam-shell conformation as the volume of oil droplet reduced to some critical value. A sequence of photographs were taken for each droplet shown and the last photograph showing a barrel shape prior to a clam-shell shape was used to extract the data point representing the transition.

Figure 3 illustrates that barrel shapes occur for large droplets, judged by the drop volume normalised by the fiber radius, or low contact angles and that clam-shell shapes occur for small droplets or high contact angles. We would argue from the global geometry of the cylindrical fiber 
shape that such behaviour is to be expected. A droplet on a curved surface should appear to be a droplet on a plane surface if the curvature of the surface is low. Starting with a barrel shaped droplet on a fiber we can imagine the radius of the cylinder being increased so that locally the fiber surface appears flat. If no transition in conformation occurs the droplet shape becomes that of a stripe and this does not correspond to the droplet shape we would expect on a plane surface. However, if we have a clam-shell droplet on a fiber and increase the radius of the cylinder so that locally the surface appears flat then we can imagine the droplet shape changing smoothly into that of a spherical-cap type droplet on a plane surface. Thus, we would expect a barrel conformation to transform into a clam-shell conformation as drop volume relative to fiber radius decreased. Roll-up is therefore a direct consequence of the global geometry of the cylindrical shape.

\section{Stability conditions and comparison to data}

Two types of stability condition can be considered. The first is absolute stability in the sense of the conformation with the lowest surface free energy for a given droplet volume and contact angle, as discussed in section 3. The second is the stability of a given shape against perturbations of the shape and this is referred to as metastability. Carroll considered the effect of a small perturbation on a barrel shaped droplet and argued that changes in the Laplace excess pressure controlled the response of the system to the perturbation [18]. He suggested that figure 3 could be separated into two regions by the locus of points satisfying the equation,

$$
2 n^{3} \cos \theta_{e}-3 n^{2}+1=0
$$

The points satisfying equation (3) are given by the lower solid curve in figure 3. According to Carroll's criterion points above this line correspond to stable barrel shaped droplets and points below this line correspond to metastable conformations possessing axial symmetry. McHale et al, re-examined this argument and demonstrated that the region above this curve can be further divided into two types as indicated by the dotted curve in figure 3 [19]. Above the dotted curve barrel shaped droplets with an inflection point in their profile occur, whereas below the dotted curve the barrel shaped droplets have no inflection in their profile. The significance of the inflection point is that the solution to equation (1) involves a parameterisation that can be double valued and two paths, both satisfying the boundary condition on the contact angle, can exist in the solution. The path involving the inflection angle gives a change in relative sign of the radii of 
curvature at the contact line with consequences on the Laplace excess pressure. It was conjectured that Carroll's criterion for stability may only be valid for barrel shaped droplets with inflection points in their profiles. The dotted curve in figure 3 is the minimum drop size for which a barrel shape droplet can have an inflection in its profile. This corresponds to the inflection angle occurring at the contact line and is given by $[8,19]$,

$$
n_{\min }=\frac{1+\sin \theta_{e}}{\cos \theta_{e}}
$$

The finite element calculations (section 3) allow, for the first time, the surface free energy of the two droplet conformations to be directly compared and, in this sense, the absolute stability to be determined. The data from the finite element calculation of absolute stability (table 2) are shown as the upper solid curve in figure 3. Regions below this curve correspond to clam-shell shaped droplets that have a surface free energy lower than that of barrel shaped droplets having the same contact angle and the same drop volume. Regions above this curve are tentatively assigned as barrel shapes with lower energy than clam-shells. The reason we qualify the region above the curve as tentatively is that more accuracy in the finite element calculation could in principle lower the computed energy of the clam-shell shapes examined in this region. Refining the finite element mesh always resulted in a slightly lower energy for the clam-shell shape. We are therefore more confident that the region below the curve does correspond to clam-shell shapes with lower energy than the equivalent barrels, i.e. the curve is a lower bound in separating the regions in figure 3.

One immediate conclusion of the energy comparison is that all barrel shaped drops that are absolutely stable also have inflections in their profiles. Moreover, the comparison between the experimental data and the stability criteria show that the final measured barrel shapes prior to barrel droplets undergoing roll-up have inflection points in their profiles. The majority, although not all, of these drops also lie above the curve indicating absolute stability. None of the experimental points lie in the region between the curve indicating metastability and the inflection point condition. This is important because McHale et al originally suggested that contact angles for the axially symmetric barrel shape could be deduced by measuring the inflection angle and one further parameter, such as the drop reduced radius (i.e. maximal radius normalized by the fiber radius) or reduced contact length (i.e. wetted length along the fiber divided by fiber radius) [8]. No previous work has presented theoretical calculations showing that stable barrel shaped drops are likely to belong to those possessing an inflection angle. The presentation of 
experimental data in figure 3, chosen by Carroll, uses the barrel drop reduced radius and the contact angle as parameters [23]. However, the original data was obtained in the form of the reduced radius and reduced contact length and the solution for the axially symmetric barrel shape used to calculate the contact angle [23]. Figure 4 presents the data and the three curves from figure 3 in this form using the programs developed by McHale et al. to convert between barrel shape parameters [8]. The scatter in the data on this diagram is reduced compared to figure 3 and all curves appear as good approximations to straight lines. The agreement between the experimental data and the curve indicating absolute stability is good, particularly when taking into account the limitations in the experimental data and allowing for the fact that all accessible experimental data must lie to the left of the theoretical line due to them being pre-critical drops [18]. To have further confidence in the difference between the stability criteria, the inflection angle condition and metastability, additional experimental data is required. It is hoped that the calculations of the clam-shell conformation will provide further motivation for new experiments.

\section{Conclusion}

The wetting of a fiber surface has been considered with a particular emphasis on the role of the global geometry of the cylindrical shape. Differences between the two conformations of droplet possible on a fiber have been discussed. A physical argument for the transition from the axially symmetric barrel conformation to the asymmetric clam-shell conformation with reducing drop volume or increasing fiber radius has been provided. Finite element calculations have been performed for the clam-shell shape droplet and the surface free energy of the shape obtained. A curve indicating the absolute stability of clam-shell droplets, for which their energy is lower than that of equivalent barrel shaped droplets, has been constructed. This curve has been compared to both experimental data and a curve indicating metastability. The relative location of the curve indicating barrel shaped droplets with an inflection angle has also been considered. It has been shown that all barrel shaped droplets that are absolutely stable also possess an inflection in their profile and that these drops are also stable according to the metastability condition. The curve of absolute stability for the axially symmetric conformation is in good agreement with literature data on roll-up.

\section{Acknowledgement}

The authors are grateful to Dr B.J. Carroll at Unilever Research, Port Sunlight, for discussions on the topic of this work. 


\section{References}

1. R.N. Wenzel, Ind. Eng. Chem., 28 (1936) 988.

2. T. Onda, S. Shibuichi, N. Satoh and K. Tsujii, Langmuir, 12 (1996) 2125.

3. D. Quéré and D. Richard, Europhys Lett., $\underline{48}$ (1999) 286.

4. T. Bieker and S. Dietrich, Physica A, 252 (1998) 85.

5. C. Bauer and S. Dietrich, Phys. Rev E., $\underline{62}$ (2000) 2428.

6. F. Brochard, J. Chem. Phys., $\underline{84}$ (1986) 4664.

7. D. Quéré, J.-M. Di Meglio and F. Brochard, Rev Phys Appl., 23 (1988) 1023.

8. G. McHale, N.A. Käb, M.I. Newton and S.M. Rowan, J. Colloid. Interf. Sci., 186 (1997) 453.

9. A. V. Neimark, J. Adhes. Sci. Technol., 13 (1999) 1137.

10. D. Quéré, Annu. Rev. Fluid Mech., 31 (1999) 347.

11. B.J. Carroll, Colloids and Surfaces A: Physicochemical and Engineering Aspects, 74 (1993) 131.

12. N. Bernet, P.E. Bourban and J.A.E. Manson, J. Thermoplastic Comp. Maters., 13 (2000) 434.

13. T.W. Ebbesen, J. Phys. Chem. Solids, 57 (1996) 951.

14. L. Léger and J. F. Joanny, Rep. Prog. Phys., 55 (1992) 431.

15. B.J. Carroll, J. Colloid Interface Sci., $\underline{9}$ (1984) 195.

16. B.J. Carroll, J. Colloid. Interf. Sci. 186 (1976) 488.

17. N.K. Adam, J. Soc. Dyers Colour, $\underline{53}$ (1937) 122.

18. B.J. Carroll, Langmuir, 2 (1986) 248.

19. G. McHale, M.I. Newton and B.J. Carroll, Oil \& Gas Sci. Technol. - Rev. IFP, $\underline{56}$ (2001) 47.

20. B.J. Carroll, B.G.C. O’Rourke and A.J.I. Ward, J. Pharm. Pharmacol., 34 (1982) 287.

21. G. McHale, et al, J. Adhes. Sci. Technol., 13 (1999) 1457.

22. K.A. Brakke (2000), Surface Evolver.www.susqu.edu/facstaff/b/brakke/evolver.

23. B.J. Carroll, personal communication, 2001. 


\section{Tables}

Table 1 Literature data for roll-up. Equilibrium contact angle, $\theta_{\mathrm{e}}$ and reduced maximal droplet thickness, $n$, were taken from ref. [18]. Reduced wetted length, $L_{\mathrm{r}}$, and volume, $V_{\mathrm{r}}$, were calculated using the programs developed in [8].

\begin{tabular}{cccc}
$\theta_{\mathrm{e}}{ }^{\mathrm{o}}$ & $V_{\mathrm{r}}=V / x_{\mathrm{f}}$ & $L_{\mathrm{r}}=L_{\mathrm{W}} / x_{\mathrm{f}}$ & $n$ \\
\hline 10.13 & 26.315 & 7.100 & 1.763 \\
14.93 & 29.299 & 6.766 & 1.836 \\
16.07 & 46.464 & 7.604 & 2.101 \\
19.06 & 48.103 & 7.399 & 2.138 \\
18.05 & 89.703 & 8.949 & 2.585 \\
22.24 & 84.822 & 8.450 & 2.560 \\
21.94 & 141.183 & 9.823 & 3.007 \\
19.96 & 207.831 & 11.118 & 3.406 \\
24.10 & 251.751 & 11.395 & 3.659 \\
25.90 & 142.009 & 9.519 & 3.043 \\
28.06 & 147.269 & 9.450 & 3.092 \\
29.80 & 200.892 & 10.233 & 3.430 \\
28.00 & 88.245 & 8.081 & 2.633 \\
27.34 & 49.148 & 6.745 & 2.210 \\
31.12 & 59.417 & 6.888 & 2.355 \\
34.05 & 43.884 & 6.009 & 2.186 \\
33.93 & 102.937 & 8.025 & 2.802 \\
36.93 & 89.979 & 7.466 & 2.705 \\
41.91 & 93.679 & 7.227 & 2.778 \\
42.15 & 327.691 & 10.888 & 4.118 \\
53.84 & 795.465 & 13.432 & 5.616 \\
61.93 & 611.879 & 11.738 & 5.217 \\
63.25 & 840.848 & 12.951 & 5.797 \\
68.59 & 1572.502 & 15.492 & 7.174
\end{tabular}


Table 2 Finite element results for the clam-shell shape. The largest volume for which a clamshell shape has a lower surface free energy than a barrel shape is shown in column two. The bracketed value indicates the next higher volume for which the energy of the clam-shell appears to be higher than that of the barrel.

\begin{tabular}{cclccc}
$\theta_{\mathrm{e}}{ }^{\mathrm{o}}$ & $V_{\mathrm{r}}=V / x_{\mathrm{f}}$ & & $F / \gamma_{\mathrm{LV}}$ & $L_{\mathrm{r}}=L_{\mathrm{W}} / x_{\mathrm{f}}$ & $n$ \\
\hline 14 & 7.9 & $(8.0)$ & 8.286 & 4.5117 & 1.382 \\
16 & 10.0 & $(10.1)$ & 10.426 & 4.682 & 1.453 \\
18 & 12.4 & $(12.5)$ & 12.832 & 4.848 & 1.527 \\
20 & 15.0 & $(15.1)$ & 15.406 & 4.997 & 1.602 \\
22 & 18.4 & $(18.5)$ & 18.642 & 5.193 & 1.689 \\
25 & 24.3 & $(24.4)$ & 24.093 & 5.471 & 1.823 \\
30 & 37.8 & $(38.0)$ & 35.781 & 5.979 & 2.076 \\
35 & 59.3 & $(59.5)$ & 52.623 & 6.606 & 2.387 \\
40 & 94 & $(95)$ & 76.871 & 7.364 & 2.769 \\
45 & 154 & $(156)$ & 113.54 & 8.333 & 3.257 \\
50 & 266 & $(267)$ & 172.06 & 9.626 & 3.910 \\
55 & 493 & $(495)$ & 270.90 & 11.412 & 4.813 \\
60 & 1010 & $(1020)$ & 452.33 & 14.017 & 6.132 \\
65 & 2400 & $(2450)$ & 827.62 & 18.130 & 8.213 \\
70 & 7200 & $(7250)$ & 1756.8 & 25.408 & 11.891
\end{tabular}




\section{Figure Captions}

Figure 1 Droplet conformations on a fiber: (a) barrel, and (b) clam-shell.

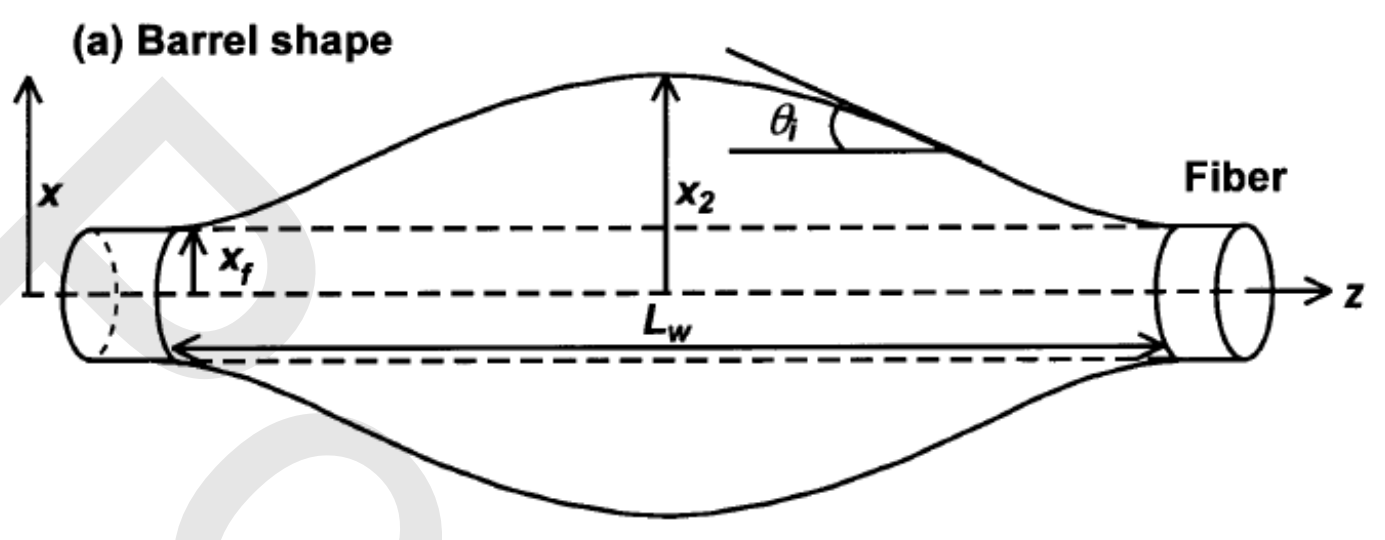

(b) Clam-shell shape

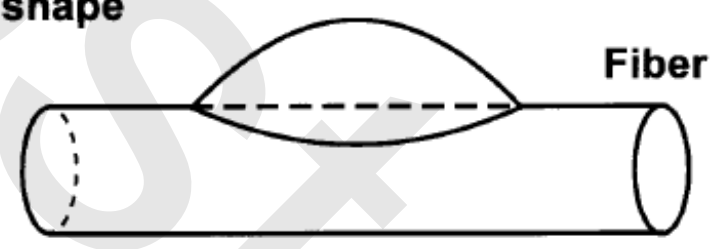

Figure 2 Axial and side views of clam-shell shapes with volumes corresponding to the absolute stability transition volumes: (a) $\theta_{\mathrm{e}}=30^{\circ}, V / x_{\mathrm{f}}{ }^{3}=37.8$, and (b) $\theta_{\mathrm{e}}=60^{\circ}, V / x_{\mathrm{f}}{ }^{3}=1010$.

(a)

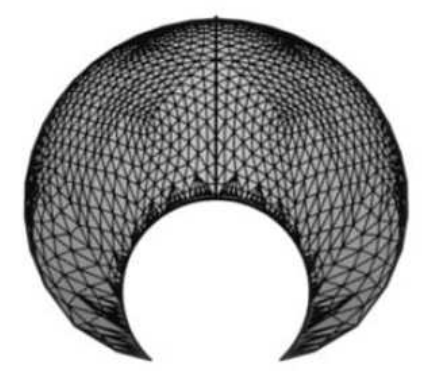

(b)
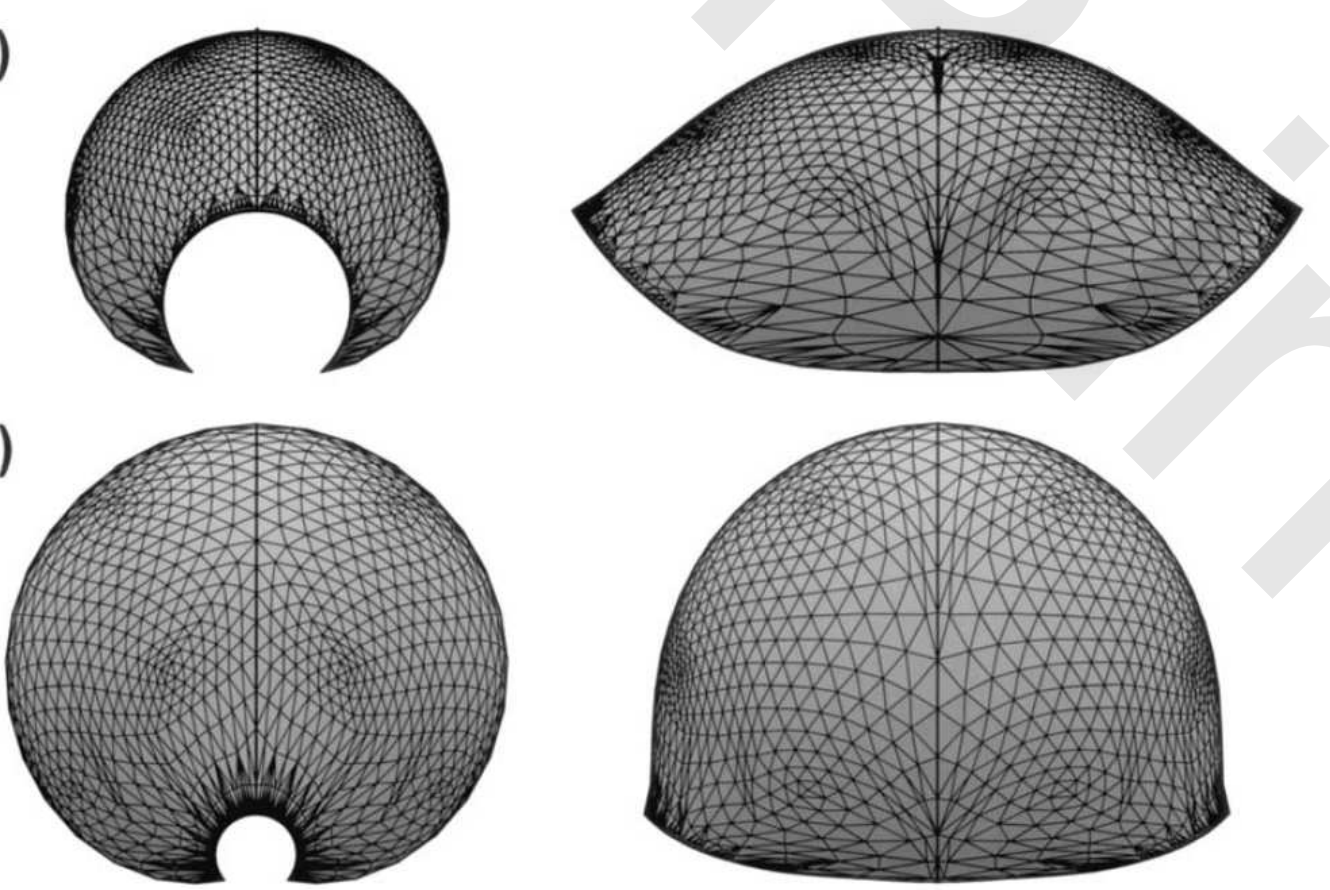

Figure 3 Literature data [18] for the barrel drop size at which roll-up occurs. The upper solid curve is the absolute stability condition, the dotted curve is the drop size at which the 
inflection point in the profile touches the fiber surface, and the lower curve is the metastability condition.

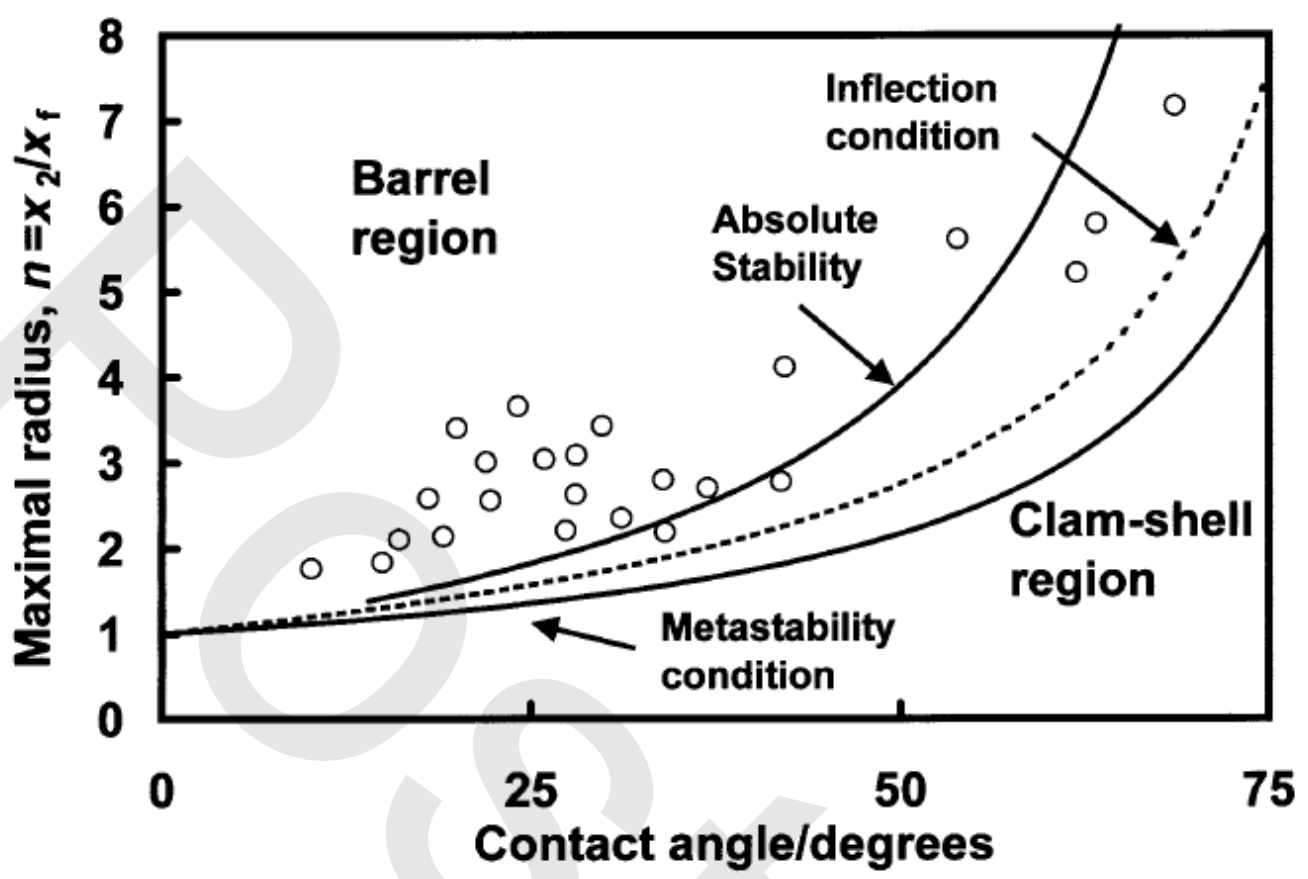

Figure 4 Data from figure 3 presented using the measured barrel shaped drop parameters of drop reduced length and reduced maximal drop radius. The curves are the same as figure 3 .



